\title{
High preoperative serum CA 19-9 levels can predict poor oncologic outcomes in colorectal cancer patients on propensity score analysis
}

\author{
Jung Kyong Shin', Hee Cheol Kim¹, Woo Yong Lee', Seong Hyeon Yun', Yong Beom Cho', Jung Wook Huh', \\ Yoon Ah Park', Ho-Kyung Chun ${ }^{2}$ \\ ${ }^{1}$ Department of Surgery, Samsung Medical Center, Sungkyunkwan University School of Medicine, Seoul, Korea \\ ${ }^{2}$ Department of Surgery, Kangbuk Samsung Hospital, Sungkyunkwan University School of Medicine, Seoul, Korea
}

\begin{abstract}
Purpose: The purpose of this study is to evaluate the prognostic value of preoperative serum CA 19-9 levels in colorectal cancer patients.

Methods: Between 2008 and 2011, 4,794 consecutive patients who underwent curative resection for colorectal cancer were analyzed. These patients were classified into 2 groups according to preoperative CA 19-9 (high CA 19-9: $\geq 37 \mathrm{ng} / \mathrm{mL}$, $\mathrm{n}=440$; normal CA 19-9: $<37 \mathrm{ng} / \mathrm{mL}, \mathrm{n}=4,354)$. We used 1:20 propensity score matching to adjust for potential baseline confounders between groups.

Results: After matching, 424 patients (10.5\%) among 4,021 patients with colorectal cancer showed a high pre-CA 19-9 level ( $\geq 37 \mathrm{ng} / \mathrm{mL}$ ). There were no significant differences between these 2 groups in age, sex, preoperative CEA level, or T, $\mathrm{N}$, and $\mathrm{M}$ stage after matching. Of the 424 patients with high pre-CA 19-9, 141 (33.3\%) exhibited cancer recurrence more frequently than patients with normal preoperative CA 19-9 (18.5\%). Patients with an elevated preoperative CA 19-9 level showed significantly poorer survival than those with normal levels. The 5 -year overall survival rate was $79.7 \%$ in the high preoperative CA $19-9$ group and $91.9 \%$ in the normal preoperative CA $19-9$ group (P < 0.001). The 5-year disease-free survival rate was $70.2 \%$ in the high preoperative CA $19-9$ group and $82.7 \%$ in the normal preoperative CA $19-9$ group $(P<$ 0.0011 .

Conclusion: Patients with an elevated preoperative CA 19-9 level in colorectal cancer have a significantly poorer prognosis than those with normal levels of CA 19-9. We therefore suggest preoperative CA 19-9 level can be used as an additional prognostic indicator of poor outcomes in colorectal cancer.

[Ann Surg Treat Res 2019;96(3):107-115]
\end{abstract}

Key Words: CA 19-9 antigen, Prognosis, Colorectal neoplasms

\section{INTRODUCTION}

Serum tumor markers including CEA and CA 19-9 are commonly used to predict prognosis in colorectal cancer patients. The American Society of Clinical Oncology (ASCO) guidelines for the use of tumor markers in colorectal cancer have recommended CEA measurement for preoperative staging and treatment planning, as well as postoperative follow-up. However, in routine practice, checking the level of CA 19-9 as a marker of colon cancer is not recommended for screening,
Received July 12, 2018, Revised September 27, 2018, Accepted October 16, 2018

Corresponding Author: Hee Cheol Kim

Department of Surgery, Samsung Medical Center, Sungkyunkwan University School of Medicine, 81 Irwon-ro, Gangnam-gu, Seoul 06351, Korea

Tel: +82-2-3410-1655, Fax: +82-2-3410-6980

E-mail: hckimcrc@gmail.com

ORCID code: https://orcid.org/0000-0002-1610-5363
Copyright (c) 2019, the Korean Surgical Society

(c) Annals of Surgical Treatment and Research is an Open Access Journal. All articles are distributed under the terms of the Creative Commons Attribution NonCommercial License (http://creativecommons.org/licenses/by-nc/4.0/) which permits unrestricted non-commercial use, distribution, and reproduction in any medium, provided the original work is properly cited. 
diagnosis, staging, or monitoring colorectal cancer [1].

CA 19-9 was described by Koprowski et al. [2] in 1979 as a monoclonal antibody against a human colorectal cancer cell line. It promotes adhesion of cancer cells to endothelial cells and plays a role in tumor progression [3,4]. CA 19-9 is often used in combination with CEA to manage colorectal cancer patients, even though it is a less sensitive tumor marker [5]. Although the ASCO guidelines suggest that there is insufficient evidence for using CA 19-9 in colorectal cancer patients [1], several studies have demonstrated the usefulness of CA 19-9 as an indicator of prognosis and recurrence [6-11].

In the present study, we sought to investigate the usefulness of preoperative serum CA 19-9 level as a prognostic factor in a large cohort of colorectal cancer patients at a tertiary hospital.

\section{METHODS}

Between January 2008 and December 2011, 4,794 consecutive patients who underwent curative resection for colorectal cancer at the Samsung Medical Center were enrolled and clinical and pathologic characteristics analyzed. We collected patient data from the prospectively managed colorectal cancer database at Samsung Medical Center. Insufficient data were re-evaluated using electronic medical charts. This study was approved by the Institutional Review Board (IRB) of Samsung Medical Center (2018-07-010-001). Written informed consent was waived by the IRB.

Serum levels of CA 19-9 and CEA were measured via chemiluminescent immunoassay in the laboratory of the same hospital. Preoperative CA 19-9 and CEA were measured, with CEA $\geq 5 \mathrm{ng} / \mathrm{mL}$ and CA $19-9 \geq 37 \mathrm{ng} / \mathrm{mL}$ regarded as elevated. Patients were divided into the normal CA 19-9 group ( $n=4,354$, $90.8 \%$ ), and the high CA $19-9$ group ( $n=440,9.2 \%$ ). In order to evaluate the oncologic effects of serum CA 19-9, we used 20:1 propensity score matching to adjust for potential baseline confounders including age, sex, preoperative CEA level, tumor location and size, cell differentiation, and pathologic T, N, and M stage, lymphatic invasion, perineural invasion, vascular invasion, and AJCC stage. After matching, a total of 4,021 patients, $3.597(89.5 \%)$ in the normal CA 19-9 group and 424 (10.5\%) for high CA 19-9 group, were analyzed.

During the follow-up period, the patients visited the outpatient clinic every 3 months for the first 2 years, every 6 months for the subsequent 3 years, and annually thereafter. Regular laboratory tests including serum CEA and CA 19-9 were performed. Chest $\mathrm{CT}$ and abdominopelvic $\mathrm{CT}$ scans were performed every 6 months or every year during the followup period. Peritoneal seeding was diagnosed intraoperatively in cases of stage IV disease, and postoperative recurrence as peritoneal carcinomatosis was diagnosed by findings during reoperation for recurrence or the presence of abnormal intraperitoneal nodules or peritoneal thickening as detected on abdominopelvic CT scans or PET CT scans.

Statistical analyses were carried out using SAS ver. 9.4 (SAS Institute Inc., Cary, NC, USA) and R3.2.2 (R Foundation for Statistical Computing, Vienna, Austria). We used KolmogorovSmirnov test for the normality test. The significance of differences between groups was evaluated using the chi-square test, 2 sampled t-test or analysis of variance, as appropriate. Survival rates were calculated using the Kaplan-Meier method, and prognostic factors and survival curves were compared using the log-rank test. A P-value of 0.05 or less was considered statistically significant.

\section{RESULTS}

\section{Clinicopathologic characteristics of the patients before and after propensity score matching}

The clinicopathological characteristics of the patients included in the study are listed in Table 1 . The median followup period was 56.1 months and 55.3 months in the normal and high CA 19-9 groups, respectively. The median age was 60 years in the normal CA 19-9 group and 59 years in the high CA 19-9 group ( $P=0.428$ ). Patients with high CA 19-9 levels had significantly higher preoperative CEA levels. Of the 440 patients with high CA 19-9 levels, 192 patients (43.6\%) also had a high preoperative serum CEA level $(\mathrm{P}<0.001)$. Patients with high $\mathrm{CA}$ 19-9 levels were also more likely to have aggressive pathologic features. The average tumor size (mean \pm standard deviation) was $4.1 \pm 2.4 \mathrm{~cm}$ in the normal CA $19-9$ group, and $5.7 \pm 2.5$ $\mathrm{cm}$ in the high CA $19-9$ group ( $\mathrm{P}<0.001$ ). Poorly differentiated/ mucinous adenocarcinoma and signet ring cell carcinoma were significantly more frequent in the high CA 19-9 group ( $\mathrm{P}<$ 0.001). Lymphatic invasion was detected in 1,312 patients $(30.1 \%)$ in the normal CA 19-9 group, and in 216 patients (49.1\%) in the high CA 19-9 group ( $P<0.001$ ). Venous invasion was observed in 718 patients (16.5\%) in the normal CA 19-9 group and in 137 patients $(31.1 \%)$ in the higher CA $19-9$ group $(\mathrm{P}<0.001)$. AJCC stage was more advanced in the high CA 19-9 group than in the normal preoperative serum CA 19-9 ( $\mathrm{P}<0.001$ ).

After propensity score matching, 424 patients among 4,021 patients (10.5\%) showed a high preoperative CA 19-9 level. There were no significant differences between the 2 groups in age, sex, preoperative CEA level, T, N, or M stage, histologic grade of differentiation, or lymphovascular/perineural invasion after propensity score matching. The clinicopathological characteristics of the matched patients are presented in Table 1.

\section{Survival according to preoperative CA 19-9 levels}

Survival analyses according to preoperative CA 19-9 levels of matched patients are shown in Fig. 1. The 5-year overall survival rate was $91.9 \%$ in the normal CA $19-9$ group and $79.7 \%$ in the 
Table 1. Patient clinicopathologic characteristics before and after propensity score matching analysis

\begin{tabular}{|c|c|c|c|c|c|c|}
\hline \multirow[b]{2}{*}{ Characteristic } & \multicolumn{3}{|c|}{ Before propensity score matching } & \multicolumn{3}{|c|}{ After propensity score matching } \\
\hline & $\begin{array}{c}\text { CA } 19-9<37 \\
(n=4,354)\end{array}$ & $\begin{array}{c}\text { CA } 19-9 \geq 37 \\
(n=440)\end{array}$ & P-value & $\begin{array}{c}\text { CA } 19-9<37 \\
(\mathrm{n}=3,597)\end{array}$ & $\begin{array}{c}\text { CA } 19-9 \geq 37 \\
(n=424)\end{array}$ & P-value \\
\hline Age (yr) & $60 \pm 11$ & $59 \pm 13$ & 0.428 & $59 \pm 12$ & $59 \pm 12$ & 0.984 \\
\hline Sex & & & $<0.001$ & & & $>0.999$ \\
\hline Male & $2,709(62.2)$ & $219(49.8)$ & & $1,850(51.4)$ & $218(51.4)$ & \\
\hline Female & $1,645(37.8)$ & $221(50.2)$ & & $1,747(48.6)$ & $206(48.6)$ & \\
\hline Preoperative CEA (ng/mL) & & & $<0.001$ & & & 0.508 \\
\hline Normal $(<5)$ & $3,761(86.4)$ & $248(56.4)$ & & $2,105(58.5)$ & $241(56.8)$ & \\
\hline High $(\geq 5)$ & $593(13.6)$ & $192(43.6)$ & & $1,492(41.5)$ & $183(43.2)$ & \\
\hline Tumor location & & & $<0.001$ & & & 0.097 \\
\hline Right colon & $949(21.8)$ & $153(34.8)$ & & 1,193 (33.2) & $144(34.0)$ & \\
\hline Left colon & $1,410(32.4)$ & $127(28.8)$ & & 1,095 (30.4) & $127(30.0)$ & \\
\hline Rectum & $1,995(45.8)$ & $160(36.4)$ & & 1,309 (36.4) & $153(36.0)$ & \\
\hline Pathologic T stage & & & $<0.001$ & & & 0.276 \\
\hline $\mathrm{T} 1, \mathrm{~T} 2$ & $1,489(34.2)$ & $39(8.9)$ & & $411(11.4)$ & $41(9.7)$ & \\
\hline $\mathrm{T} 3, \mathrm{~T} 4 \mathrm{a}, \mathrm{b}$ & $2,865(65.8)$ & $401(91.1)$ & & $3,186(88.6)$ & $383(90.3)$ & \\
\hline Pathologic $N$ stage & & & $<0.001$ & & & 0.658 \\
\hline No & $2,449(56.2)$ & $143(32.5)$ & & 1,295 (36.0) & 148 (34.9) & \\
\hline $\mathrm{N} 1,2$ & 1,905 (43.8) & $297(67.5)$ & & $2,302(64.0)$ & $276(65.1)$ & \\
\hline Pathologic $M$ stage & & & $<0.001$ & & & 0.852 \\
\hline MO & 4,152 (95.6) & $356(79.5)$ & & $2,991(83.1)$ & $351(82.8)$ & \\
\hline M1a, M1b & $194(4.4)$ & $92(20.5)$ & & $606(16.9)$ & $73(17.2)$ & \\
\hline Size of tumor $(\mathrm{cm})$ & $4.1 \pm 2.4$ & $5.7 \pm 2.5$ & $<0.001$ & $5.4 \pm 2.5$ & $5.6 \pm 2.3$ & 0.097 \\
\hline Cell differentiation & & & $<0.001$ & & & 0.549 \\
\hline$W D+M D$ & 4,025 (92.4) & $358(81.4)$ & & $3,035(84.4)$ & $353(83.3)$ & \\
\hline $\mathrm{PD}+\mathrm{MUC}+\mathrm{SRC}$ & $329(7.6)$ & $82(18.6)$ & & $562(15.6)$ & $71(16.7)$ & \\
\hline Lymphatic invasion & & & $<0.001$ & & & 0.831 \\
\hline$(+)$ & $1,312(30.1)$ & $216(49.1)$ & & $1,669(46.4)$ & $199(46.9)$ & \\
\hline$(-)$ & 3,042 (69.9) & $224(50.9)$ & & 1,928 (53.6) & $225(53.1)$ & \\
\hline Venous invasion & & & $<0.001$ & & & 0.536 \\
\hline$(+)$ & $718(16.5)$ & $137(31.1)$ & & $1,017(28.3)$ & $126(30.0)$ & \\
\hline$(-)$ & $3,636(83.5)$ & $303(68.9)$ & & $2,580(71.7)$ & $298(70.0)$ & \\
\hline Perineural invasion & & & $<0.001$ & & & 0.757 \\
\hline$(+)$ & $532(12.2)$ & $112(25.5)$ & & 849 (23.6) & $103(24.3)$ & \\
\hline$(-)$ & $3,822(87.8)$ & $328(74.5)$ & & $2,748(76.4)$ & $321(75.7)$ & \\
\hline AJCC stage & & & $<0.001$ & & & 0.808 \\
\hline I & $1,206(27.7)$ & $30(6.8)$ & & $314(8.7)$ & $31(7.3)$ & \\
\hline II & 1,303 (29.9) & 104 (23.6) & & $901(25.0)$ & 107 (25.2) & \\
\hline III & 1,649 (37.9) & $216(49.1)$ & & $1,776(49.4)$ & $213(50.3)$ & \\
\hline IV & $196(4.5)$ & $90(20.5)$ & & $606(16.9)$ & $73(17.2)$ & \\
\hline
\end{tabular}

Values are presented as mean \pm standard deviation or number $(\%)$.

WD, well-differentiated; MD, moderately differentiated; PD, poorly differentiated; MUC, mucinous adenocarcinoma; SRC, signet ring cell carcinoma; AJCC, American Joint Committee on Cancer.

high CA 19-9 group ( $\mathrm{P}<0.001)$. Patients with high preoperative CA 19-9 levels tended to show a decrease in the survival rate for all stages compared to those with normal levels, but this was not significant for stage I patients (Fig. 2). The 5-year diseasefree survival rate was $82.7 \%$ in the normal CA $19-9$ group and
$70.2 \%$ in high CA $19-9$ group ( $\mathrm{P}<0.001$ ). The 5-year diseasespecific survival rate was also significantly different between the 2 groups ( $93.5 \%$ vs. $82.9 \%, \mathrm{P}<0.001)$. High preoperative CA 19-9 levels were significantly associated with poor prognosis for stages III and IV (Fig. 3). 

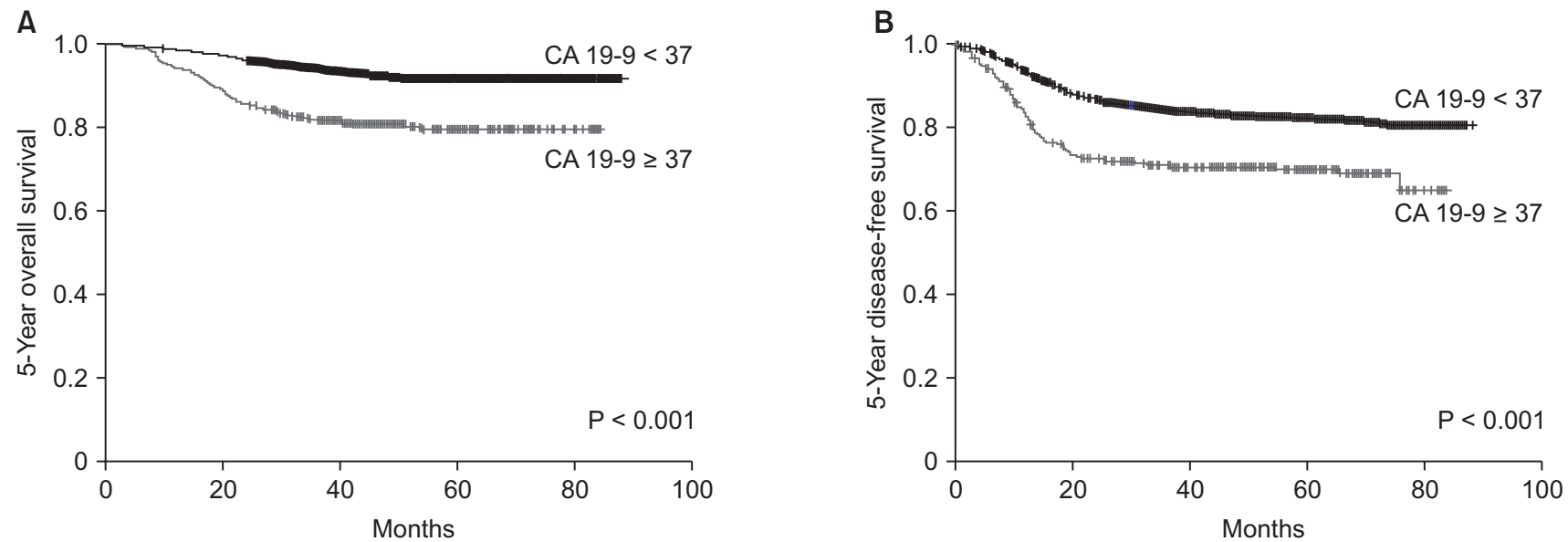

Fig. 1. Five-year survival curves stratified by preoperative serum CA 19-9 level in matched cohorts. Five-year overall survival curves (A) and 5-year disease-free survival curves (B).
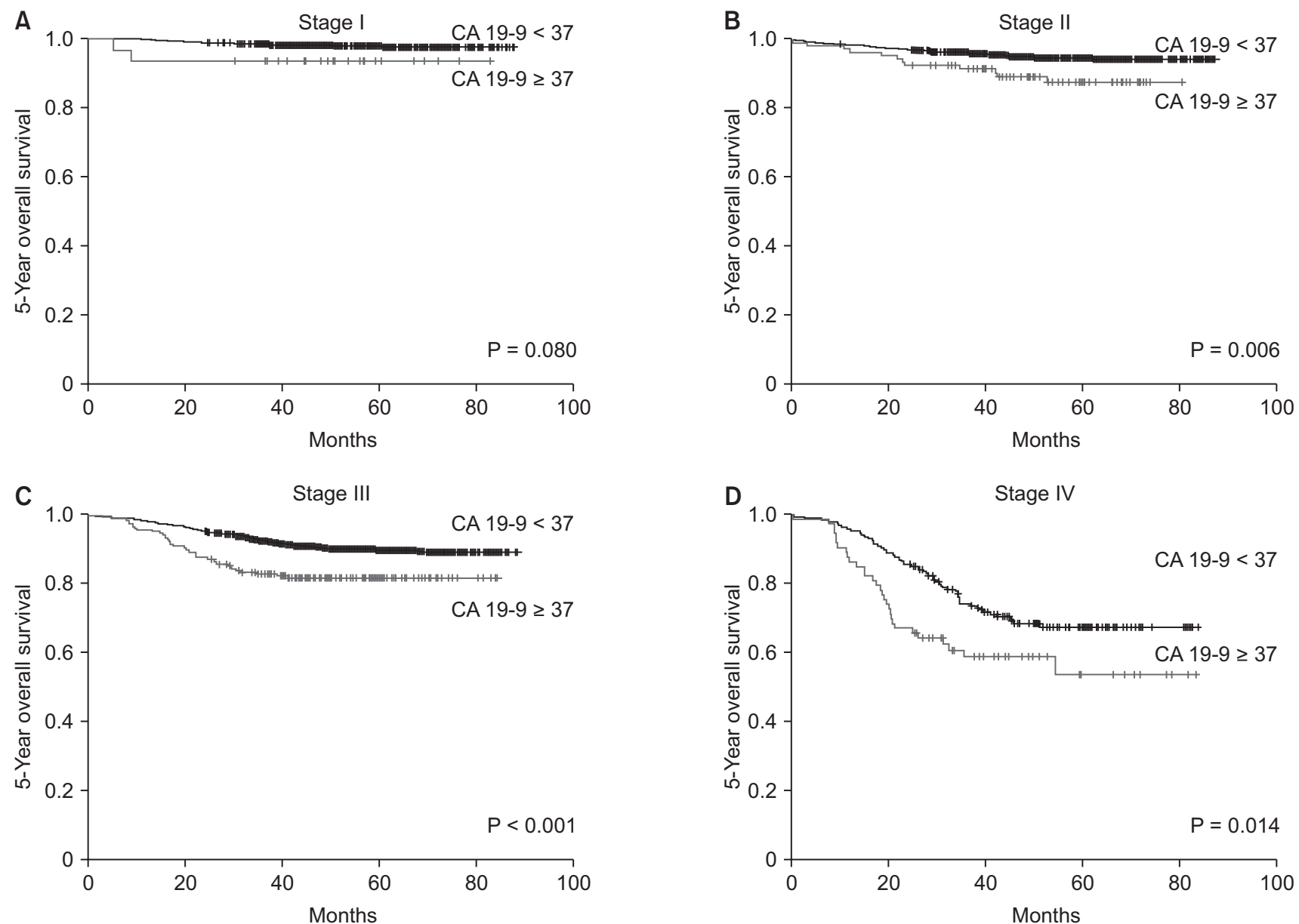

Fig. 2. Five-year overall survival and preoperative serum CA 19-9 levels in matched patients with stage I (A), stage II (B), stage III (C), and stage IV (D) diseases.

Analysis of the prematching cohort showed similar results. The 5 -year overall survival rate was $92.9 \%$ in the normal CA 19-9 group and $77.7 \%$ in the high CA $19-9$ group ( $<<0.001$ ). The 5 -year disease-free survival rate was $83.1 \%$ and $64.8 \%$ in the normal and high CA 19-9 groups, respectively $(\mathrm{P}<0.001$ ). The 5-year disease-specific survival rate was also significantly different between the 2 groups $(95.9 \%$ vs. $85.0 \%, \mathrm{P}<0.001)$. 

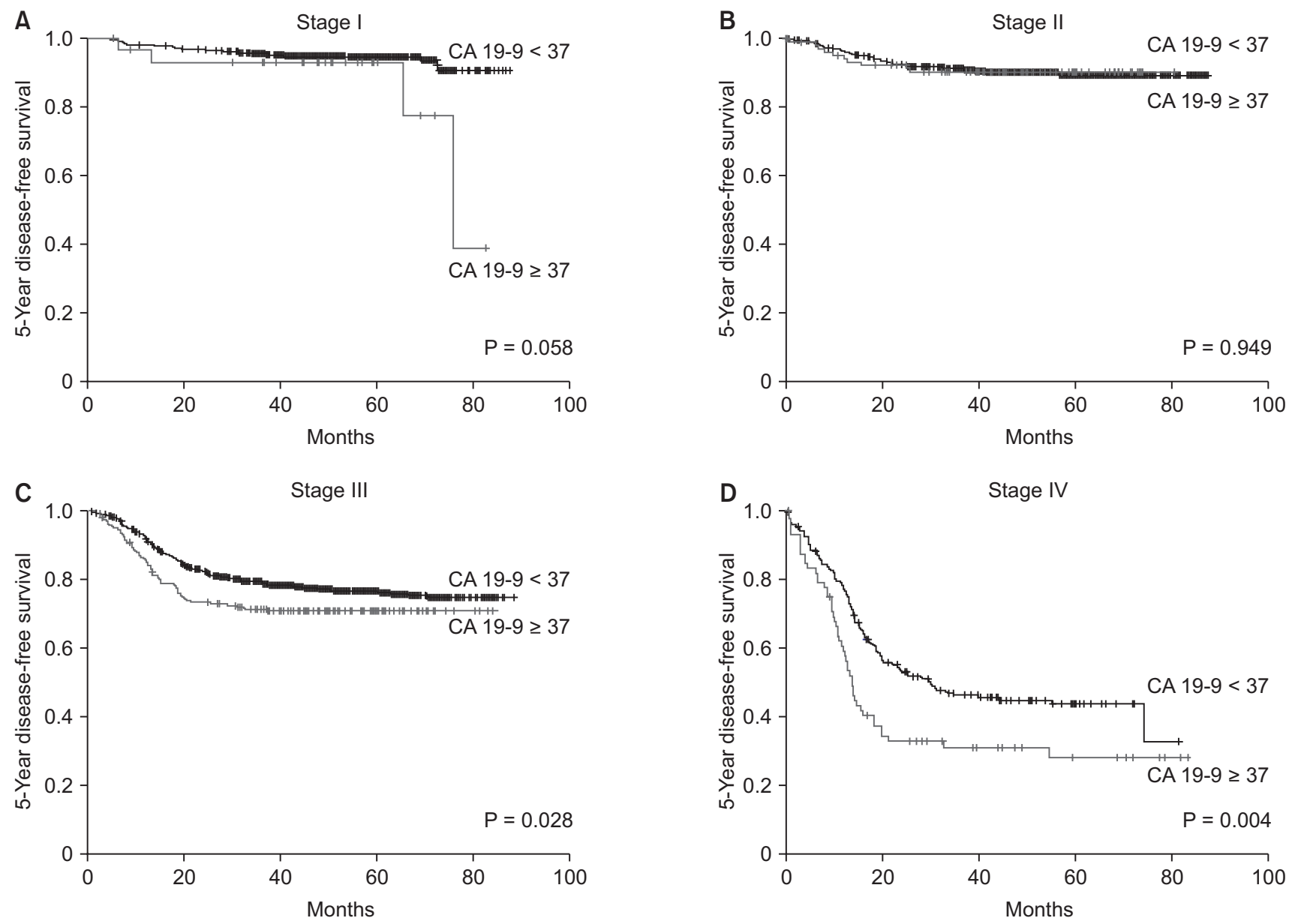

Fig. 3. Five-year disease-free survival and preoperative serum CA 19-9 levels in matched patients with stage I (A), stage II (B), stage III (C), and stage IV (D) diseases.
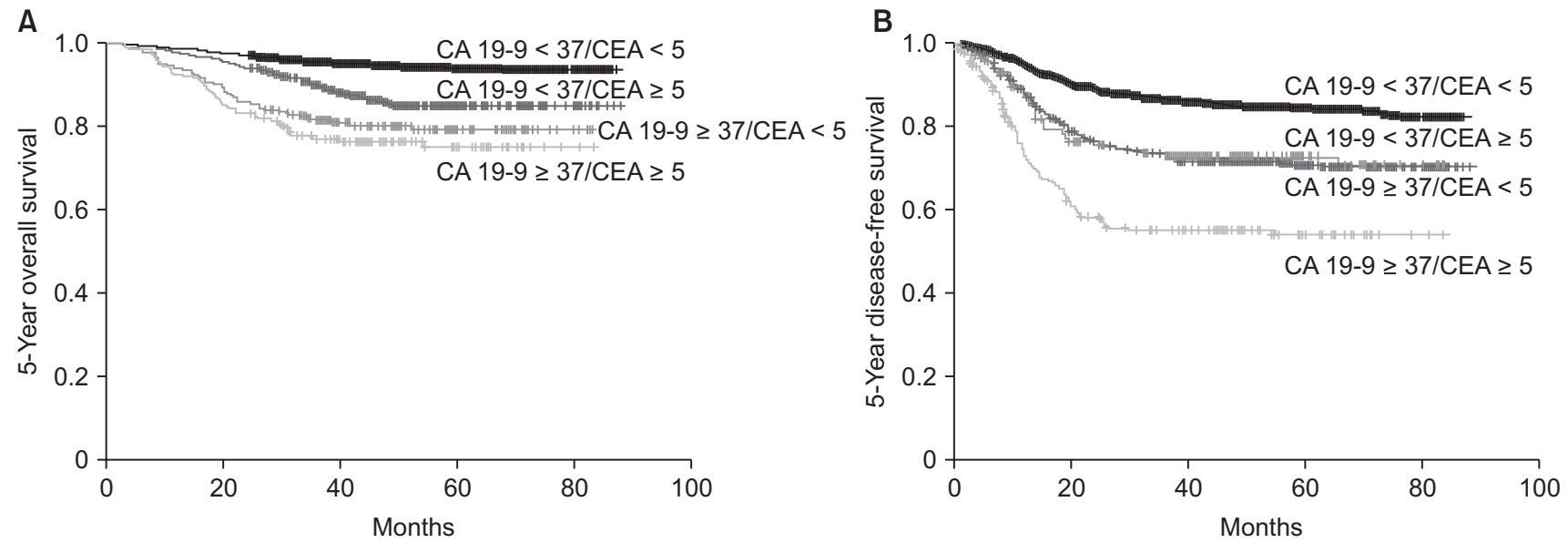

Fig. 4. Five-year overall survival and disease-free survival curves stratified by preoperative serum CA 19-9 and CEA levels in matched cohorts.

\section{Survival according to preoperative CA 19-9 and} CEA levels after propensity score matching Survival analysis according to preoperative CA 19-9 and CEA levels is shown in Fig. 4. The 5-year overall survival rate was $94.1 \%$ in patients with normal results for both measures, 85.2\% in patients with elevated CEA and normal CA 19-9 (P < 0.001), and $79.6 \%$ in patients with elevated CA $19-9$ and normal CEA $(\mathrm{P}<0.001)$. Patients with a preoperative increase in only CA 19-9 
had significantly decreased 5-year overall survival compared with patients with a preoperative increase of only CEA $(79.6 \%$ vs. $85.2 \%, P=0.048)$. The 5 -year disease-free survival rate was $84.9 \%$ in patients with normal CEA and CA $19-9,71.2 \%$ in patients with elevated CEA and normal CA 19-9 ( $P<0.001)$, and $72.9 \%$ in patients with elevated CA $19-9$ and normal CEA (P < $0.001)$. However, there was no significant difference in 5 -year disease-free survival between patients with a preoperative increase only one of the two measures (CA 19-9 vs. CEA, 72.9\% vs. $71.2 \%, \mathrm{P}=0.932$ ).

We analyzed fiver-year overall survival and disease-free survival with stages I, II, III, and IV disease. In stage IV, the 5-year overall survival rate was $45.0 \%$ in patients with normal CEA and elevated CA $19-9,67.5 \%$ in patients with elevated CEA and normal CA 19-9 $(P=0.006)$. There was no significant difference in 5-year disease free survival and overall survival in stages I, II, and III.

\section{Prognostic factors for DFS and OS}

Univariate and multivariate analyses were performed to evaluate the value of CA 19-9 elevation as an independent prognostic factor with respect to overall survival and diseasefree survival. On univariate analysis (Table 2), factors associated with poorer overall survival included age $<60$ years, high preoperative CA 19-9 and CEA levels, lymphovascular and perineural invasion, pathologic T3 or T4 category, pathologic $\mathrm{N} 1$ or N2 category, and poorly differentiated/mucinous adenocarcinoma or signet ring cell carcinoma. In multivariate analysis age, high CA 19-9 and CEA levels, lymphovascular invasion, pathologic $\mathrm{T}$ and $\mathrm{N}$ stage, and histologic differentiation were associated with poorer overall survival.

Over a median follow-up period of 56 months, colorectal cancer recurred in 806 patients (665 patients with normal CA 19-9 levels, 141 patients with high CA 19-9 levels). Factors associated with poorer disease-free survival on univariate analysis (Table 3) included high preoperative CA 19-9 and CEA levels, lymphovascular and perineural invasion, pathologic T3 or T4 disease, pathologic N1 or N2 disease, and poorly differentiated or mucinous adenocarcinoma or signet ring cell carcinoma. In multivariate analysis, high CA 19-9 and CEA levels, lymphovascular invasion, pathologic $\mathrm{T}$ and $\mathrm{N}$ stage, and histologic differentiation were associated with poorer diseasefree survival.

\section{Patterns of recurrence according to preoperative CA 19-9 levels}

Of the 4,021 colorectal cancer patients who underwent curative primary tumor resection, 806 patients $(20.0 \%)$ showed recurrence during the follow-up period. Recurrence was observed in 141 patients (33.3\%) with elevated preoperative CA 19-9 levels and in 665 patients (18.5\%) with normal CA 19-9 levels $(P=0.001)$. With regard to the recurrence site, patients with high CA 19-9 levels showed significantly higher rates of peritoneal carcinomatosis (18.4\%) than patients with normal CA 19-9 levels (7.1\%) $(\mathrm{P}<0.001)$ (Table 4).

Table 2. Univariate and multivariate analysis of risk factors for overall survival (OS) after surgery in matched cohort

\begin{tabular}{|c|c|c|c|c|}
\hline \multirow{2}{*}{ Variable } & \multicolumn{2}{|c|}{ Univariate } & \multicolumn{2}{|c|}{ Multivariate } \\
\hline & $\mathrm{HR}(95 \% \mathrm{Cl})$ & P-value & $\mathrm{HR}(95 \% \mathrm{Cl})$ & P-value \\
\hline Age, $>60 \mathrm{yr}$ & $1.987(1.598-2.471)$ & $<0.001$ & $2.017(1.621-2.510)$ & $<0.001$ \\
\hline Male sex & $0.850(0.688-1.050)$ & 0.131 & - & - \\
\hline CA $19-9, \geq 37$ ng/mL & $2.820(2.204-3.610)$ & $<0.001$ & $1.944(1.502-2.517)$ & $<0.001$ \\
\hline $\mathrm{CEA},>5 \mathrm{ng} / \mathrm{mL}$ & $2.053(1.648-2.558)$ & $<0.001$ & $1.304(1.036-1.642)$ & 0.024 \\
\hline Lymphatic invasion & $2.510(2.037-3.094)$ & $<0.001$ & $1.541(1.216-1.953)$ & $<0.001$ \\
\hline Vascular invasion & 3.239 (2.626-3.997) & $<0.001$ & $2.062(1.638-2.595)$ & $<0.001$ \\
\hline Perineural invasion & $2.162(1.708-2.738)$ & $<0.001$ & $1.211(0.940-1.561)$ & 0.139 \\
\hline \multicolumn{5}{|c|}{ Histologic differentiation } \\
\hline $\mathrm{PD}+\mathrm{MUC}+\mathrm{SRC}$ & $2.764(2.142-3.566)$ & $<0.001$ & $2.019(1.558-2.615)$ & $<0.001$ \\
\hline \multicolumn{5}{|l|}{ pT category } \\
\hline $\mathrm{T} 3+\mathrm{T} 4$ & $3.791(2.577-5.576)$ & $<0.001$ & $2.068(1.379-3.101)$ & $<0.001$ \\
\hline \multicolumn{5}{|l|}{ pN category } \\
\hline $\mathrm{N}$ positive & $2.816(2.235-3.548)$ & $<0.001$ & $1.397(1.060-1.841)$ & 0.017 \\
\hline \multicolumn{5}{|l|}{ pM category } \\
\hline M positive & $3.489(2.694-4.519)$ & $<0.001$ & $5.652(4.437-7.199)$ & $<0.001$ \\
\hline
\end{tabular}

$\mathrm{HR}$, hazard ratio; $\mathrm{Cl}$, confidence interval; $\mathrm{PD}$, poorly differentiated; MUC, mucinous adenocarcinoma; SRC, signet ring cell carcinoma. 
Table 3. Univariate and multivariate analysis of risk factors for disease-free survival after surgery in matched cohort

\begin{tabular}{|c|c|c|c|c|}
\hline \multirow{2}{*}{ Variable } & \multicolumn{2}{|c|}{ Univariate } & \multicolumn{2}{|c|}{ Multivariate } \\
\hline & $\mathrm{HR}(95 \% \mathrm{Cl})$ & P-value & $\mathrm{HR}(95 \% \mathrm{Cl})$ & P-value \\
\hline Age, $>60 \mathrm{yr}$ & $1.041(0.900-1.203)$ & 0.588 & - & - \\
\hline Male sex & 1.147 (0.990-1.329) & 0.067 & - & - \\
\hline CA $19-9, \geq 37$ ng/mL & $1.996(1.646-2.422)$ & $<0.001$ & $1.366(1.118-1.669)$ & 0.002 \\
\hline CEA, $\geq 5 \mathrm{ng} / \mathrm{mL}$ & $1.859(1.588-2.176)$ & $<0.001$ & $1.282(1.087-1.512)$ & 0.003 \\
\hline Lymphatic invasion & $2.278(1.970-2.634)$ & $<0.001$ & $1.194(1.013-1.407)$ & 0.034 \\
\hline Vascular invasion & $2.954(2.544-3.429)$ & $<0.001$ & $1.694(1.439-1.995)$ & $<0.001$ \\
\hline Perineural invasion & $2.994(2.561-3.501)$ & $<0.001$ & $1.727(1.457-2.047)$ & $<0.001$ \\
\hline \multicolumn{5}{|c|}{ Histologic differentiation } \\
\hline$P D+M U C+S R C$ & $1.540(1.241-1.910)$ & $<0.001$ & $1.256(1.009-1.563)$ & 0.041 \\
\hline \multicolumn{5}{|l|}{ pT category } \\
\hline $\mathrm{T} 3+\mathrm{T} 4$ & $3.173(2.484-4.054)$ & $<0.001$ & $1.693(1.305-2.196)$ & $<0.001$ \\
\hline \multicolumn{5}{|l|}{$\mathrm{pN}$ category } \\
\hline $\mathrm{N}$ positive & $3.377(2.862-3.986)$ & $<0.001$ & $1.993(1.641-2.419)$ & $<0.001$ \\
\hline \multicolumn{5}{|l|}{ pM category } \\
\hline M positive & $2.956(2.434-3.590)$ & - & $5.558(4.641-6.657)$ & $<0.001$ \\
\hline
\end{tabular}

$\mathrm{HR}$, hazard ratio; $\mathrm{Cl}$, confidence interval; $\mathrm{PD}$, poorly differentiated; $\mathrm{MUC}$, mucinous adenocarcinoma; SRC, signet ring cell carcinoma.

Table 4. Long-term oncologic outcomes of matched cohorts

\begin{tabular}{lccr}
\hline \multicolumn{1}{c}{ Variable } & $\begin{array}{c}\text { CA 19-9: } \\
\text { normal } \\
(\mathrm{n}=3,597)\end{array}$ & $\begin{array}{c}\text { CA 19-9: } \\
\text { high } \\
(\mathrm{n}=424)\end{array}$ & P-value \\
& $\begin{array}{ccc} \\
\text { Follow-up period (mo) }\end{array}$ & $\begin{array}{c}56.1 \pm 22.7 \\
55.3 \pm 22.4\end{array}$ & 0.855 \\
Recurrent & $665(18.5)$ & $141(33.3)$ & 0.001 \\
Recurrence site & & & \\
$\quad$ Locoregional & $68(10.2)$ & $12(8.5)$ & 0.536 \\
$\quad$ Liver & $183(27.5)$ & $30(21.3)$ & 0.127 \\
Lung & $227(34.1)$ & $36(25.5)$ & 0.048 \\
Peritoneal seeding & $47(7.1)$ & $26(18.4)$ & $<0.001$ \\
$\quad$ Distant lymph node & $69(10.4)$ & $17(12.1)$ & 0.557 \\
$\quad$ Multiple sites & $43(6.5)$ & $14(9.9)$ & 0.145 \\
Other (ovary, brain, & $28(4.2)$ & $6(4.3)$ & 0.981 \\
$\quad$ bone) & & & \\
\end{tabular}

Values are presented as mean \pm standard deviation or number $(\%)$.

\section{DISCUSSION}

In the present study of 4,021 patients with colorectal cancer, high preoperative serum CA 19-9 level was a marker of poor prognosis. Both serum CEA and CA 19-9 levels have been used in the past as prognostic markers in colorectal cancer. CEA is listed as an independent prognostic factor for colorectal cancer in several consensus treatment guidelines [1,12]. Preoperative CEA is an important independent variable in predicting prognostic outcome [13,14]; adding it to the staging system has even been considered [8]. In contrast, the clinical significance of pre-
CA 19-9 remains controversial.

This propensity score matching study was designed to compare patients with high preoperative CA 19-9 levels to those with normal preoperative CA 19-9. Our study showed that high preoperative serum CA 19-9 levels were prognostic factors for colorectal cancer. This finding is in agreement with previous results indicating that high preoperative CA 19-9 is a marker of poor prognosis [8,15-17]. Nakagoe et al. [5] reported that high preoperative serum CA 19-9 was an independent predictor of subsequent cancer recurrence in patients with node-negative colorectal cancer treated with curative resection. In contrast, several studies have reported contradictory results $[18,19]$. CA 19-9 had no predictive superiority over CEA in colorectal cancer patients in a study by Morita et al. [19], who suggested that CA 19-9 could not be used to predict prognosis or detect recurrence of colorectal cancer.

However, most previous studies enrolled small number of patients. Our study analyzed a large number of colorectal cancer patients in a single institution; moreover, most of the enrolled patients were followed for more than 3 years. Although this study was retrospective in design, the data were collected prospectively. Moreover, the propensity score matching was performed on the initial cohort in order to adjust for any difference in average outcomes. In this study, we found that a high preoperative serum CA 19-9 level was an independent marker of poor prognosis in patients with colorectal cancer with a predictive value as strong as preoperative serum CEA.

Preoperative CA 19-9 was found to be significantly associated with 5-year DFS and OS in advanced (stages III and 
IV) colorectal cancer. Preoperative CA 19-9 levels were not prognostic in patients with stage I disease, which may have been because not only the small number of early-stage patients in our study, but also the recurrence rates in cancers detected at the early stage were much lower than those detected in the advanced stage. Thus, CA 19-9 levels may also be related to tumor stage, and may therefore be relatively less effective for predicting outcomes in early-stage disease. Based on this study, patients with high CA 19-9 levels at advanced stages may benefit from risk-adapted follow-up surveillance, which can also facilitate clinical decision-making regarding adjuvant therapy.

We also showed that preoperative elevation of both CEA and CA 19-9 was an independent prognostic indicator. An elevated preoperative serum CA 19-9 level was significantly associated with both decreased 5-year overall survival and disease-free survival. Multivariate analysis also indicated that a high preoperative serum CA 19-9 level was an independent prognostic factor of poor DFS and OS. Although new molecular markers are typically focused on predicting clinical outcomes in colorectal cancer patients, our results suggest that serum elevation of CA 19-9 can provide useful clinicopathologic and oncologic information in colorectal cancer.

The present study also demonstrated that an elevated preoperative CA 19-9 level was predictive of peritoneal seeding. There were 26 patients (18.4\%) with confirmed peritoneal seeding among the 141 recurrent colorectal cancer cases with high a preoperative CA 19-9 level. Schott et al. [20] demonstrated that isolated tumor cells in the peritoneal cavity of colorectal cancer bound to CA 19-9 monoclonal antibodies at a very high frequency. Yang et al. [21] reported that the mechanism of peritoneal seeding is generally thought to involve free tumor cells from the primary tumor, but some might come from other sources, such as lymph nodes, the liver, or ovaries. They suggested that tumor invasion into peritoneal mesothelial cells might stimulate the expression of CA 19-9, or tumor cells with expression of CA 19-9 might have higher behavior ability of peritoneal metastasis pathway. Based on these previous studies as well as the findings of the present study, we speculate that colorectal cancer patients with high
CA 19-9 levels might have a higher likelihood of peritoneal carcinomatosis. Patients with peritoneal carcinomatosis usually exhibit a poorer disease course. Kerscher et al. [22] reported that despite improvements in overall outcome with prolonged median survival in the patient cohort with colorectal cancer, those with peritoneal carcinomatosis have not shown the same benefit. This might be one of the reasons why patients with high CA 19-9 showed poorer overall survival and inferior disease-free survival compared with normal CA 19-9 patients in our study.

The cumulative rates of 5 -year overall survival and diseasefree survival were significantly lower in the high CA 19-9 group than in the normal CA 19-9 group. Positive CA 19-9 expression on tumor tissue, and elevated pre and postoperative serum levels were associated with poor survival rates [15,23]. Our study showed a significant difference in 5-year and disease-free survival curves between elevated serum CA 19-9 and normal CA 19-9 patients. Moreover, patients with preoperative CA 19$9^{+} /$CEA l$^{-}$levels showed poorer 5 -year survival compared with patients with CA 19-9/CEA ${ }^{+}$levels. We suggest that the tumor marker CA 19-9 could be used to monitor disease progression in colorectal cancer patients without an increase in CEA levels.

This study had several limitations associated with its retrospective nature and single-center design. Subgroup analysis of the patients in terms of perioperative treatment and the effects of chemotherapy or radiation therapy was not performed. And we did not analyze the change of both of the tumor marker levels during the course of the disease.

In conclusion, this study suggests that preoperative serum CA 19-9 level may be a prognostic marker for colorectal cancer and could provide important information regarding peritoneal seeding. Moreover, selection of aggressive follow-up protocols based on preoperative serum CA 19-9 levels might improve patient outcomes.

\section{CONFLICTS OF INTEREST}

No potential conflict of interest relevant to this article was reported.

\section{REFERENCES}

1. Locker GY, Hamilton S, Harris J, Jessup JM, Kemeny N, Macdonald JS, et al. ASCO 2006 update of recommendations for the use of tumor markers in gastrointestinal cancer. J Clin Oncol 2006;24:5313-27.

2. Koprowski H, Steplewski Z, Mitchell K,
Herlyn M, Herlyn D, Fuhrer P. Colorectal carcinoma antigens detected by hybridoma antibodies. Somatic Cell Genet 1979;5: 957-71.

3. Zheng CX, Zhan WH, Zhao JZ, Zheng D, Wang DP, He YL, et al. The prognostic value of preoperative serum levels of CEA, CA 19-9 and CA72-4 in patients with colorectal cancer. World J Gastroenterol 2001;7:431-4.

4. Thomas WM, Robertson JF, Price MR, Hardcastle JD. Failure of CA 19-9 to detect 
asymptomatic colorectal carcinoma. Br J Cancer 1991;63:975-6

5. Nakagoe T, Sawai T, Tsuji T, Jibiki MA, Nanashima A, Yamaguchi H, et al. Preoperative serum level of CA $19-9$ predicts recurrence after curative surgery in nodenegative colorectal cancer patients. Hepatogastroenterology 2003;50:696-9.

6. Zhang LN, OuYang PY, Xiao WW, Yu X, You KY, Zeng ZF, et al. Elevated CA 19-9 as the most significant prognostic factor in locally advanced rectal cancer following neoadjuvant chemoradiotherapy. Medicine (Baltimore) 2015;94:e1793.

7. Kouri M, Pyrhonen S, Kuusela P. Elevated CA 19-9 as the most significant prognostic factor in advanced colorectal carcinoma. J Surg Oncol 1992;49:78-85.

8. Chen CC, Yang SH, Lin JK, Lin TC, Chen WS, Jiang JK, et al. Is it reasonable to add preoperative serum level of CEA and CA 19-9 to staging for colorectal cancer? J Surg Res 2005;124:169-74.

9. Takakura Y, Ikeda S, Imaoka Y, Urushihara T, Itamoto T. An elevated preoperative serum carbohydrate antigen 19-9 level is a significant predictor for peritoneal dissemination and poor survival in colorectal cancer. Colorectal Dis 2015;17:41725.

10. Barillari P, Bolognese A, Chirletti P, Cardi M, Sammartino P, Stipa V. Role of CEA, TPA, and Ca 19-9 in the early detection of localized and diffuse recurrent rectal cancer. Dis Colon Rectum 1992;35:471-6.
11. Ueda T, Shimada E, Urakawa T. The clinicopathologic features of serum CA 199-positive colorectal cancers. Surg Today 1994;24:518-25.

12. Yu H, Son GM, Joh YG. The clinical significance of preoperative serum levels of carbohydrate antigen 19-9 in colorectal cancer. J Korean Surg Soc 2013;84:231-7.

13. Huh JW, Lee WY, Park YA, Cho YB, Yun $\mathrm{SH}, \mathrm{Kim} \mathrm{HC}$, et al. Prognostic factors associated with primary cancer in curatively resected stage IV colorectal cancer. J Cancer Res Clin Oncol 2014;140:435-41.

14. Park IJ, Choi GS, Lim KH, Kang BM, Jun SH. Serum carcinoembryonic antigen monitoring after curative resection for colorectal cancer: clinical significance of the preoperative level. Ann Surg Oncol 2009;16:3087-93.

15. Nakayama T, Watanabe M, Teramoto T, Kitajima M. CA 19-9 as a predictor of recurrence in patients with colorectal cancer. J Surg Oncol 1997;66:238-43.

16. Lin PC, Lin JK, Lin CC, Wang HS, Yang $\mathrm{SH}$, Jiang JK, et al. Carbohydrate antigen $19-9$ is a valuable prognostic factor in colorectal cancer patients with normal levels of carcinoembryonic antigen and may help predict lung metastasis. Int J Colorectal Dis 2012;27:1333-8.

17. Reiter W, Stieber P, Reuter C, Nagel D, LauWerner U, Lamerz R. Multivariate analysis of the prognostic value of CEA and CA 19-9 serum levels in colorectal cancer. Anticancer Res 2000;20(6D):5195-8.
18. Yakabe T, Nakafusa Y, Sumi K, Miyoshi A, Kitajima Y, Sato S, et al. Clinical significance of CEA and CA 19-9 in postoperative follow-up of colorectal cancer. Ann Surg Oncol 2010;17:2349-56.

19. Morita S, Nomura T, Fukushima Y, Morimoto T, Hiraoka N, Shibata N. Does serum CA 19-9 play a practical role in the management of patients with colorectal cancer? Dis Colon Rectum 2004:47:227-32.

20. Schott A, Vogel I, Krueger U, Kalthoff H, Schreiber HW, Schmiegel W, et al. Isolated tumor cells are frequently detectable in the peritoneal cavity of gastric and colorectal cancer patients and serve as a new prognostic marker. Ann Surg 1998; 227:372-9.

21. Yang SH, Lin JK, Lai CR, Chen CC, Li AF, Liang WY, et al. Risk factors for peritoneal dissemination of colorectal cancer. J Surg Oncol 2004:87:167-73.

22. Kerscher AG, Chua TC, Gasser M, Maeder U, Kunzmann V, Isbert C, et al. Impact of peritoneal carcinomatosis in the disease history of colorectal cancer management: a longitudinal experience of 2406 patients over two decades. Br J Cancer 2013;108: 1432-9.

23. Shimono R, Mori M, Akazawa K, Adachi Y, Sgimachi K. Immunohistochemical expression of carbohydrate antigen 19-9 in colorectal carcinoma. Am J Gastroenterol 1994;89:101-5. 